\title{
A Robotized Drilling System for Rocky Wall Consolidation
}

\author{
Rezia M. Molfino, Roberto P. Razzoli, Matteo Zoppi
}

\begin{abstract}
The paper proposes a new highly automated drilling system able to create holes up to $20 \mathrm{~m}$ depth in rocky walls using standard $1.5 \mathrm{~m}$ length rods. The drilling system, to be used to automate rocky walls consolidation, has to be positioned in the points of the map earlier defined by the geologist; for this reason it is hosted onto a semiautonomous climbing platform, with rods stored onboard. An automatic system is also required to feed the drilling head with new rods while the hole progresses and to recover the rods once the hole is up. The drilling system mainly consists of: a commercial drilling rig with the requested modifications for the interfacing to an automatic feeding system; a manipulator (endowed with a suitable gripper) for the loading/unloading of the rods; a storage buffer for allocating the rods. In the paper, the alternatives considered for the design of the whole drilling system are shortly recalled, explaining the guidelines which led to the final architecture, as well.
\end{abstract}

Index Terms-Automation, Construction Robotics, Drilling system, Remote handling

\section{INTRODUCTION}

$\mathrm{T}$ raditionally rocky wall consolidation requires an operator to place manually the drilling bits in the drilling machine spindle, because the automatic feeding systems available on the market are not capable of working horizontally with small diameters rods. Furthermore, deep drilling requires skilled operators as several parameters have to be monitored in order to efficiently drive the machine and avoid mechanical damages. Today, descriptive logs of the drilling process heavily rely on visual observations that are subjective and prone to human bias, sometimes resulting in different descriptions, between loggers, of the same material extracted during the drilling.

The replacing of humans with machines in the field of

This work was partially funded by the European Commission, under Grant G1ST-CT-2002-50160, that is here greatly acknowledged.

Rezia M. Molfino, is with the Department of Mechanics and Machine Design, University of Genova, Via All'Opera Pia 15A, 16145 - Genova, Italy (corresponding author phone: +39 010 3532842; fax: +39 010 3532298; email:molfino@dimec.unige.it).

Roberto P. Razzoli is with the Department of Mechanics and Machine Design, University of Genova, Via All'Opera Pia 15A, 16145 - Genova, Italy (e-mail: razzoli@dimec.unige.it).

Matteo Zoppi is with the Department of Mechanics and Machine Design, University of Genova, Via All'Opera Pia 15A, 16145 - Genova, Italy (e-mail: zoppi@dimec.unige.it). consolidation presents several positive aspects; first of all, operators are not subjected to health's danger for the generated dust and vibrations and to accidents due to the risk of falling down or rocks impacts; then, tasks are performed all the same way and more quickly.

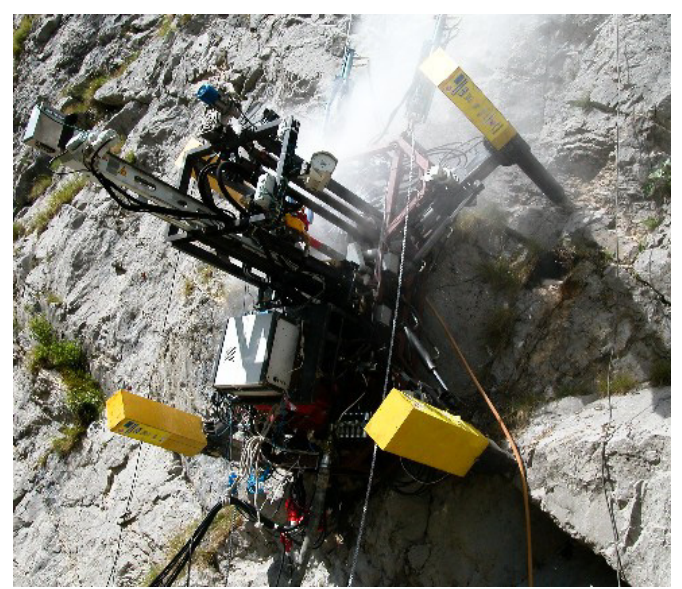

Fig. 1. Roboclimber at work (Udine, Italy, July 2004)

A novel drilling system has so been conceived to be put on board the robot called Roboclimber, [1]-[11], a 1.3 tons vehicle, Fig. 1 and Fig. 2, for climbing slopes till $85^{\circ}$ thanks to the coordinated action of its four legs, moved by hydraulic jacks, and of two lifting devices putting ropes in tension, the Tirfor ${ }^{\circledR}$.

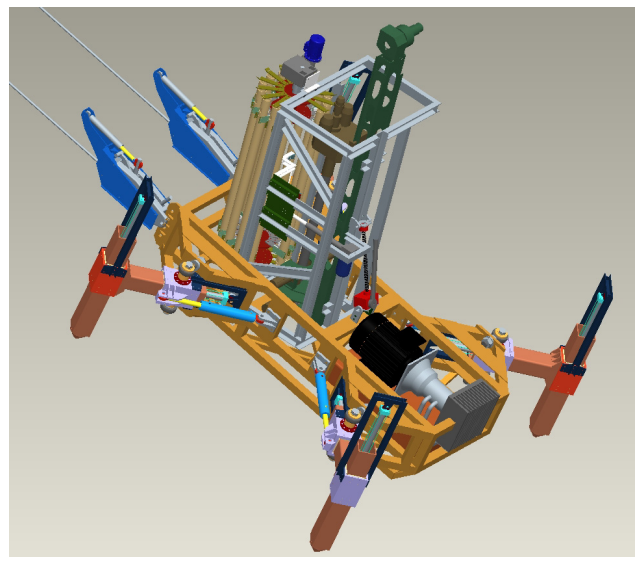

Fig. 2. Roboclimber's solid model. 
The drilling-head, the manipulator and the buffer are rigidly connected by a frame and can tilt, Fig. 3 and Fig. 4, as a whole: this reduces the required mobility of the manipulator, as the rods are always parallel to the spindle of the drilling head; the slope of the frame is set, before of the mission start, by means of a manual screw type jack.
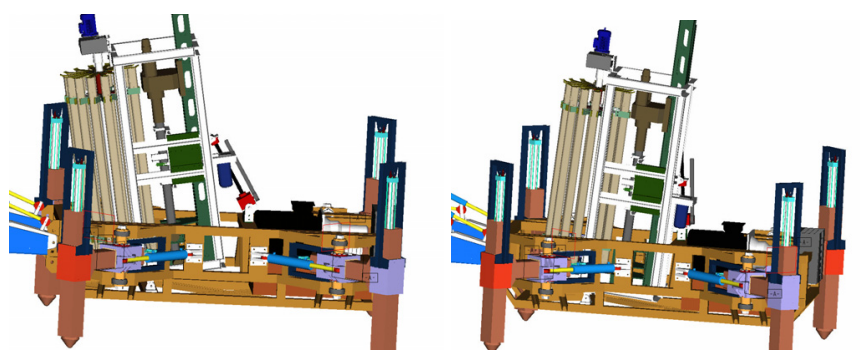

Fig. 3. Drilling frame may be tilted manually from $-5^{\circ}$ to $10^{\circ}$.

The modularity approach used along the design phases of the drilling system allows an easy reconfiguration and upgrading of the same.

The storage buffer has been designed to host the required number of rods within the minimum space: the final asset was singled out among several feasible alternatives, preferring the simplest architecture granting reliability under varying sloping conditions and firmly holding up the rods even in occurrence of impulsive loads or shocks.

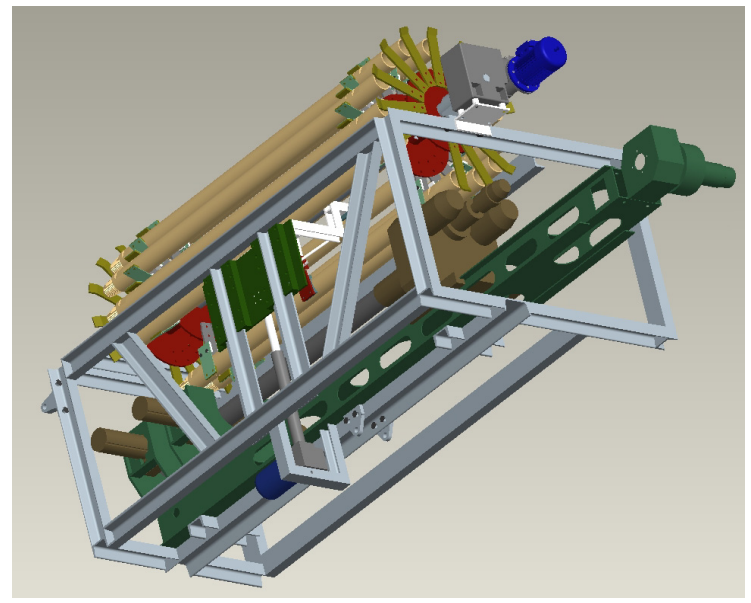

Fig. 4. Drilling frame containing the main devices: drilling unit, buffer, manipulator for rods translation.

High accuracy of the manipulator and high repeatability of the rods buffer positioning avoid misalignments of rods which might hinder the correct screwing up operations (to the spindle of the drilling head and to the other rods just inserted in the rock) and the later storage back inside the buffer. Different architectures of the manipulator were examined, considering multiple requirements as low cost, simplicity, high reliability, easy maintenance, accurate rods handling and positioning.

\section{DRILLING UNIT}

\section{A. Drilling machine specifications}

For the drilling, a modified off-the-shelf Comacchio unit (model MC 200) was used, powered by a separate Comacchio diesel portable hydraulic power unit. The hydraulic drill rig has the following technical data: $\max$ torque: $2400 \mathrm{Nm}$; $\max$ speed: $100 \mathrm{rpm}$; feed stroke: $1200 \mathrm{~mm}$; feed force: $12000 \mathrm{~N}$; retract force: $12000 \mathrm{~N}$; engine power: $27.5 \mathrm{~kW}$; mass: 1050 $\mathrm{kg}$.

The rig was equipped with a Numa Mission reverse circulation downhole hammer and a $92 \mathrm{~mm}$ drill-bit with carbide inserts. The drilling fluid is air provided by a Compair Holman 400-170S portable compressor with a nominal flow rate of $12 \mathrm{~nm}^{3} / \mathrm{min}$ at a nominal pressure of $12 \mathrm{bar}$.

The rig has been instrumented using a set of Jean Lutz sensors for the real time monitoring of the drilling parameters providing the system's status. Such data can be used to warn the remote operator about possible criticisms during drilling, and provide useful information about soil conditions. The following parameters were monitored: depth below rock surface, instantaneous advance speed, thrust, torque, rotation speed, vibration, inclination, air pressure and air flow rate.

\section{B. Power supply}

Three sources of power are needed for the drilling operations. Pneumatic: compressed air (at 12-20 bar) for the drilling unit (for the operations of drilling and flushing). Hydraulic: oil (at 200 bar) for the drilling head (to rotate and advance), and other services. Electric: for sensors, control system, cameras (for visual monitoring) and lighting.

After evaluating different solutions, it was decided that pneumatic power is generated on ground by a compressor and supplied to the robot through an umbilical cable; hydraulic power is generated on-board using a $380 \mathrm{~V}$ electric pump; electric power is generated at ground and supplied through an umbilical cable at $380 \mathrm{~V}$ : voltage can be varied on-board using transformers.

\section{BUFFER FOR RODS STORAGE}

The first layout for rods storage was a cage with properly sloped rails (Fig. 5): the rods reach the location for the manipulator's grasp thanks to gravity effect. This solution was judged unreliable and discarded because free rolling of rods along rails may be stopped by pieces of rocks or dust, and because, when drilling is made vertically, gravity's useful component for moving rods vanishes.

So, the design was addressed to revolving buffers; from the requirements about the maximum hole depth $(20 \mathrm{~m})$, a buffer containing 20 rods $1 \mathrm{~m}$ length has been initially studied.

Due to the limited room available for the buffer, the rods are placed on two coaxial circumferences; this implies having two different pick points (one per each circumference) and a major complexity of the manipulator. 


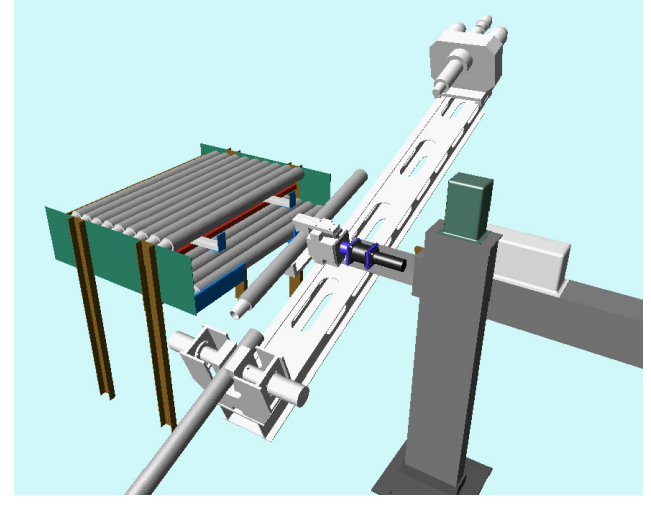

Fig. 5. First idea for the buffer: a rack with slanted rails for exploiting gravity acceleration. Note the gripper holding on a rod.

The idea was, then, to use $1.5 \mathrm{~m}$ length rods (Fig. 6): in this way fewer rods are necessary (13 rods) and they may be placed on a single circumference, simplifying their fixing and picking and reducing the number of operations per drilled hole, Fig. 7.

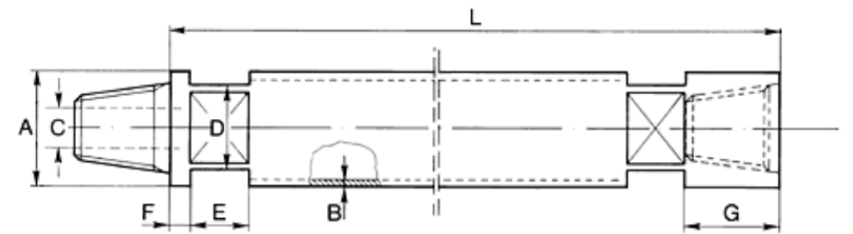

\begin{tabular}{|c|c|c|c|c|c|c|c|c|c|}
\hline $\begin{array}{c}\mathrm{A} \\
{[\mathrm{mm}]}\end{array}$ & $\begin{array}{c}\mathrm{L} \\
{[\mathrm{mm}]}\end{array}$ & $\begin{array}{c}\mathrm{B} \\
{[\mathrm{mm}]}\end{array}$ & $\begin{array}{c}\mathrm{C} \\
{[\mathrm{mm}]}\end{array}$ & $\begin{array}{c}\mathrm{D} \\
{[\mathrm{mm}]}\end{array}$ & $\begin{array}{c}\mathrm{E} \\
{[\mathrm{mm}]}\end{array}$ & $\begin{array}{c}\mathrm{F} \\
{[\mathrm{mm}]}\end{array}$ & $\begin{array}{c}\mathrm{G} \\
{[\mathrm{mm}]}\end{array}$ & $\begin{array}{c}\text { Weight } \\
{[\mathrm{kg}]}\end{array}$ & Thread \\
\hline 76 & 1500 & 6.3 & 30 & 65 & 40 & 25 & 65 & 23 & $23 / 8^{\prime \prime} \mathrm{API}$ \\
\hline
\end{tabular}

Fig. 6. Dimensions of adopted drilling rod.

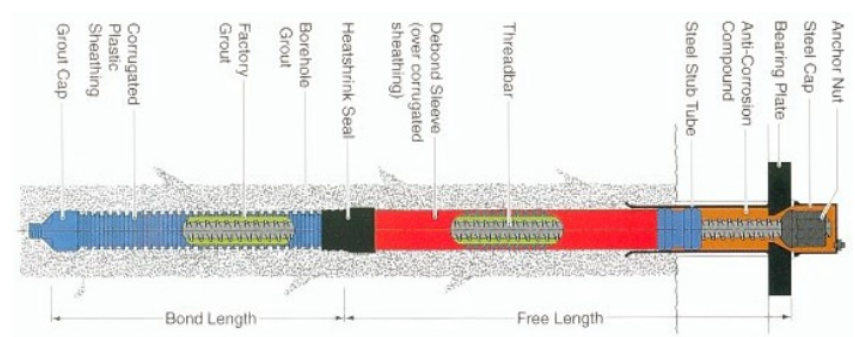

Fig. 7. Permanent bar anchors are inserted in the hole for firming-up.

The drawbacks of this solution are that a larger drilling unit and a longer hosting cage are needed, increasing the inertia and the distance of the centre of mass of the drilling system respect the wall. This has been considered in the design of the mobile platform of the robot. In the following, concepts about the buffer design are briefly recalled from the earlier version to the final one.

\section{A. Buffer with holding plugs}

In this solution, Fig. 8, two parallel plates are connected to a shaft receiving the motion by an electric motor.

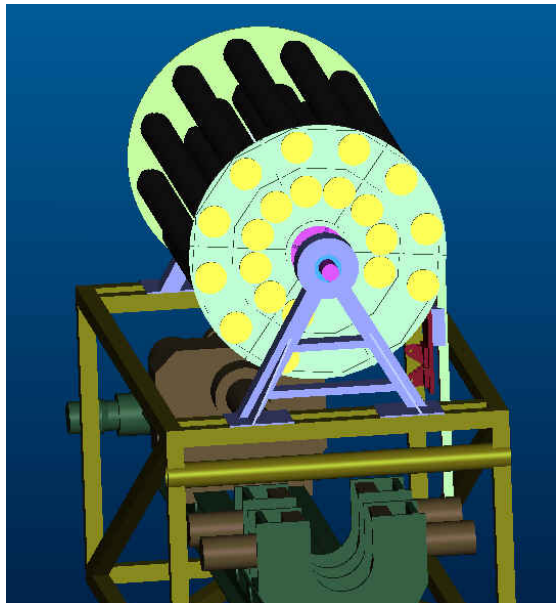

Fig. 8. The buffer mounted on the frame. The lifting device for plugs is omitted. Note the empty sector needed for allowing the gripper to have room for maneuvering when the buffer is full.

The shaft is supported by housings connected to the main cage. In the plates, holes on two circumferences are provided. The rods, having a length of $1 \mathrm{~m}$, are so arranged in two coaxial patterns to reduce the diameter of the buffer. The support of a rod is made through plugs that are pressed by springs in the hollow ends of the rod; because the ends of the rod have different size, one plate has holes (and plugs) bigger than the other one. The release of a rod, when it has reached the position over the axis of the drilling slide, is performed by acting at the same time on the two plugs holding the rod: two actuators simply lift the head of the plugs through forks when the rod is in the right position for the grip. Of course, prior the plugs moving starts, the manipulator has to firmly clamp the rod.

\section{B. Buffer with elastic sockets}

This solution presents rods placed on one circumference. Two disks have contoured springs radially placed, Fig. 9. To avoid axial movement of the rods two additional plates are appropriate, and, to prevent falling down risk, two rings are mounted, with openings near the area in which the rod is picked by the manipulator.
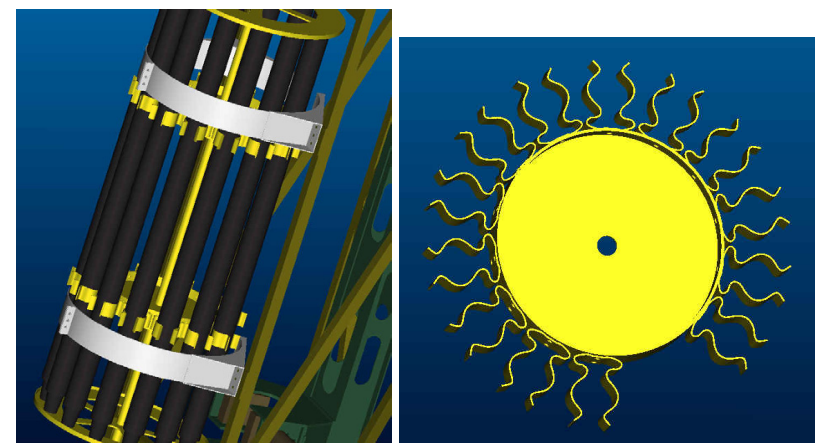

Fig. 9. On the left, a view of the buffer: outer rings, although not necessary, increase safety. On the right, one disk endowed with clips.

To release the rods, the manipulator wins the elastic force of 
the springs; because the displacement from the rest position to the position corresponding to free rod is quite high, the rings are not required to be in contact with the rods. Note that in this case a coaxial double allocation for the rod is impracticable.

\section{Buffer with open rings}

The buffer consists of two disks having 13 cuts for receiving the rods, regularly distributed, except a circular sector necessary for manipulator positioning, Fig. 10.

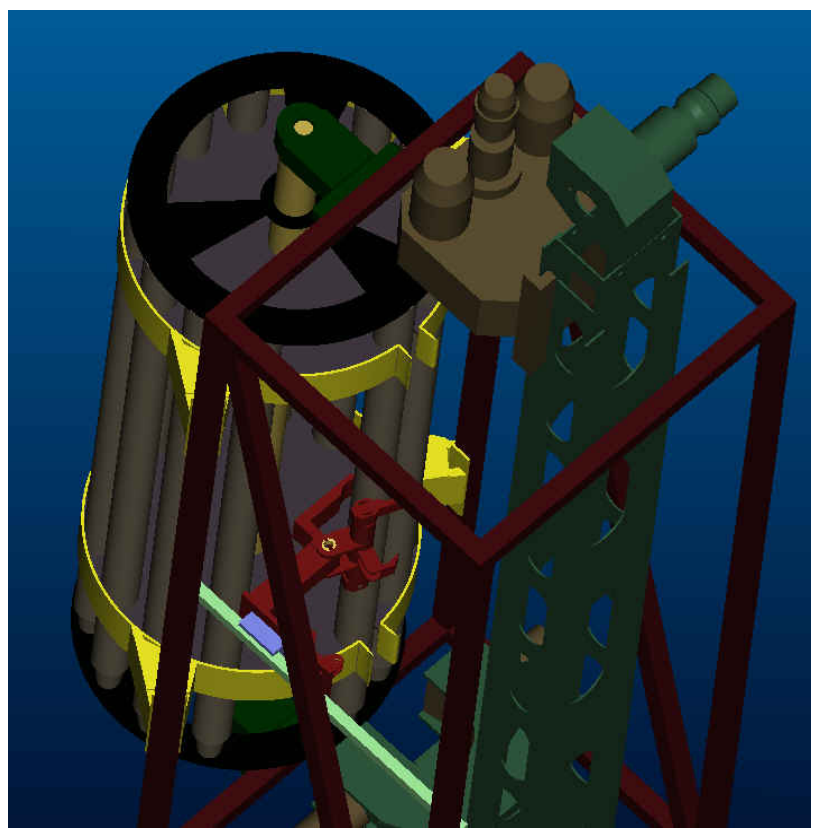

Fig. 10. Buffer with open rings.

Every disk rotates inside a ring fixed to the supporting frame.

The upper part of each ring must be locked, but the lower one has a cut where rods go through, helped by a tapered access. The opening is located over the drilling machine and has a size smaller than the rod diameter in order to avoid rod exit when no force is applied: therefore the rod passage occurs only following the manipulator action (and consequent deformation of the ring).

A Teflon ${ }^{\circledR}$ layer is applied on the inner surface to reduce friction between rods and ring.

Two additional plates are added to avoid rods axial movement.

\section{Buffer with modular leaf spring (adopted)}

The ultimate buffer is a cylindrical unit, Fig. 11, rotating at very low speed $(1 \mathrm{rpm})$, by an asynchronous $380 \mathrm{~V}$ electric motor (power: $120 \mathrm{~W}$ ) joined to a reducer with gear ratio $i=1500$.

The buffer hosts 13 rods $73 \mathrm{~mm}$ in diameter and $18 \mathrm{~kg}$ mass each; the whole capacity of the buffer is not exploited, as an angular sector of $60^{\circ}$ width has to be set free to avoid the manipulator hits some rod in the loading/unloading operations, Fig. 12.

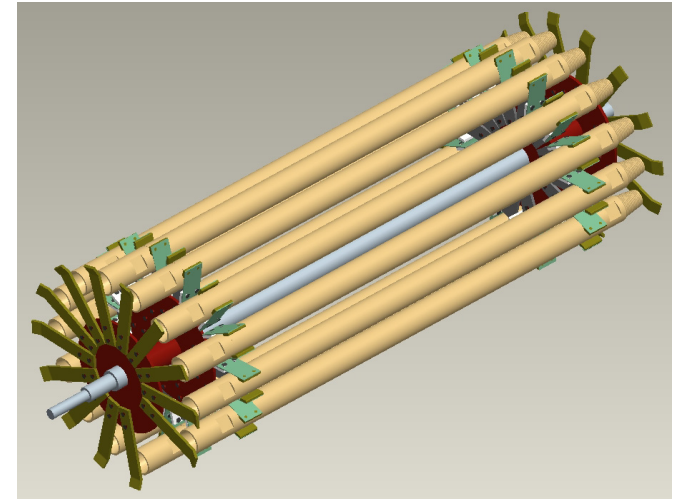

Fig. 11. A view of the revolving buffer. The supporting shaft, the hubs, springs and axial supports are visible.

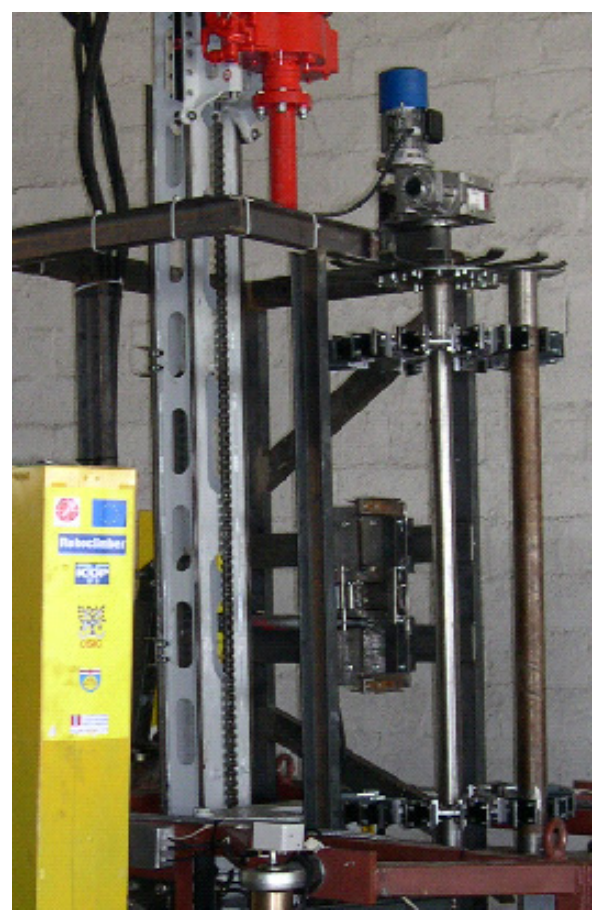

Fig. 12. A photograph taken during assembling of the Roboclimber at ICOP's shopfloor. Note the buffer with one rod mounted on.

The buffer consists of a steel shaft (diameter: $54 \mathrm{~mm}$, length: $1980 \mathrm{~mm}$ ) connected to the moto-reducer. The shaft is supported by two bearings fixed to the drilling cage and upholds two hubs on which both the plates for rods' axial constraining and the leaf spring are screwed (see Fig. 13).

\section{RODS ANCHORING SYSTEMS}

The systems for holding up the rods inside the buffer may be actuated or not actuated. For actuated solutions, a chance is using properly shaped plugs endowed with compression springs; the plugs enter the hollow ends of the rods (pushed by the springs) and release the rods once actuated; this solution is quite expensive and unreliable (an actuator could fail and the manipulator try to pick a locked rod). 

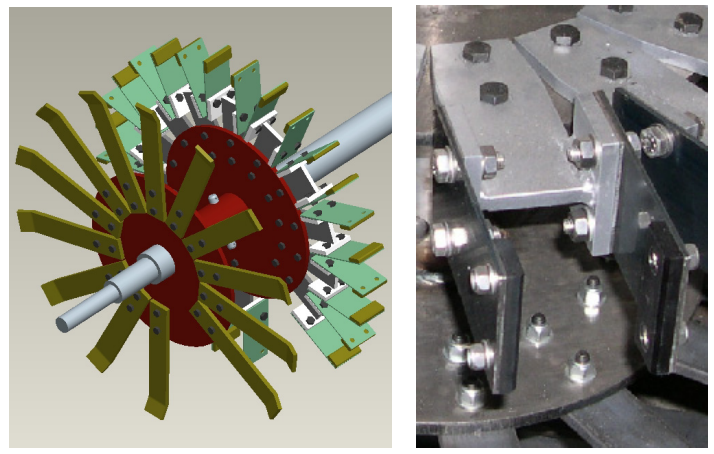

Fig. 13. The shaft of the buffer has two hubs: here one of them is shown, jointly with axial constraints and leaf springs. On the right, modular springs for rods fixing: all the parts are screwed for easy maintenance and stiffness/distance quick adjusting.

For non-actuated solutions, balls and springs, cams, clips, leaf springs, have been analyzed. Balls and springs: two different spheres are required as the holes at the ends of the rods have different diameter $(30 \mathrm{~mm}$ and $60 \mathrm{~mm})$; this implies the rod hits first the bigger ball and then the smaller with misalignment problems; furthermore, a ring giving a sufficient preload to the springs in all the positions except the grip ones might be adopted. Cams: cams assure the rods are rightly let loose, but they present difficulties in profile generation; in addition, rod loosing is made during buffer motion with the risk the rod might fall before the manipulator firmly grasps it. Clips in harmonic steel: specially shaped springs are put inside hosting sockets of the buffer disks. Rods are secured thanks to the elastic force of the deformed clips, whereas their releasing simply requires a thrust strong enough to overcome the elastic grip. Leaf plane springs: they are conceptually similar to clips and have been adopted for the prototype. The holding system of a rod consist of two pair of harmonic steel leaves (1 mm thick) screwed to a support and equipped with plastic $\left(\right.$ Ertalon $\left.{ }^{\circledR}\right)$ blocks at one extremity leaving a gap narrower than the rod diameter, Fig. 14.

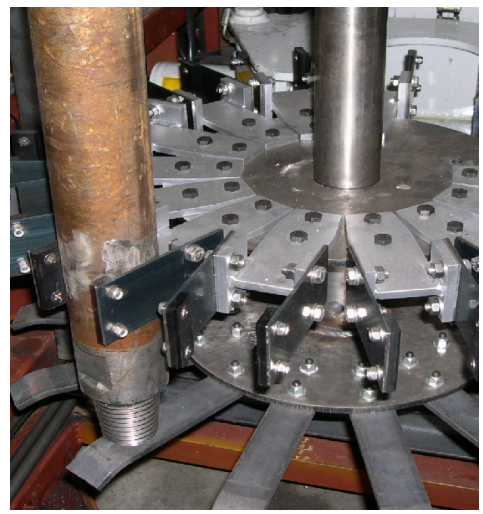

Fig. 14. Detail of how a rod is held on: bent plates constrain rod's axial motion, while leaf springs hinder radial freedom. Plates have a curved end to facilitate rods guiding as they are inserted in the buffer.

To load a rod into the buffer, the manipulator pushes it against the gap between the blocks which displaces for elastic deformation of the leaf plane springs. The advantage of this solution is the absence of actuated locking/unlocking devices, with simple design and easy manufacturing. Further, these springs, being modular, possess a good adaptability; if one should need to vary the spring stiffness, has simply to add/ remove leaves or insert/eliminate the calibrated sheets between the leaves and their support: the latter option consents to manage rods having different diameter, as well.

\section{RODS MANIPULATOR}

Since one of the major requirements for Roboclimber is to reduce human intervention, especially during drilling, it is necessary that the manipulator has characteristics for obtaining high reliability during operations. As already mentioned, rods have to be handled with high precision to avoid misalignments which might hinder their right insertion onto the drilling axis and the subsequent placing back of them inside the buffer.

Four solutions have been examined for the manipulator, with 3 and 2 DOF.

\section{A. Manipulators with $3 \mathrm{DOF}$}

A first manipulator has one rotational mobility actuated by two hydraulic cylinders arranged in sequence (to get three angular positions corresponding to their on/off states) and one translation mobility provided by a couple of parallel hydraulic cylinders acting, at the same time, as guide rails. The rod is grasped by two under-actuated hands having two fingers each. The fingers can slide inside the hand case in order to avoid collisions with the rods during the positioning operations of the manipulator. The fingers push the rod against the palm so that the positioning of the rod is accurate, the grasp is safe and the grasping force is constant for rod diameters near the nominal diameter, Fig. 15.

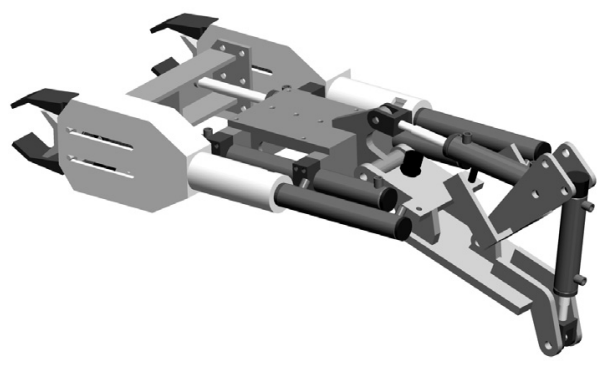

Fig. 15. First 3 DOF manipulator with serially arranged cylinders.

The alternative solution has an open structure to allow the manipulator rotate around the anchor point linked to the drilling machine. Because two cylinders are adopted, no prismatic guides are necessary for the gripper translation, since they act as guiding rails, Fig. 16.

To use a simple on-off control for the jacks, the extreme position sets are to be exploited. In its shorter configuration the jack places the rod on the drilling machine axis, while, when fully extended it is in the position related to the 
pick/store of rods in the buffer. Such manipulator architecture is also feasible to serve a buffer with rods placed on two circumferences instead of one.
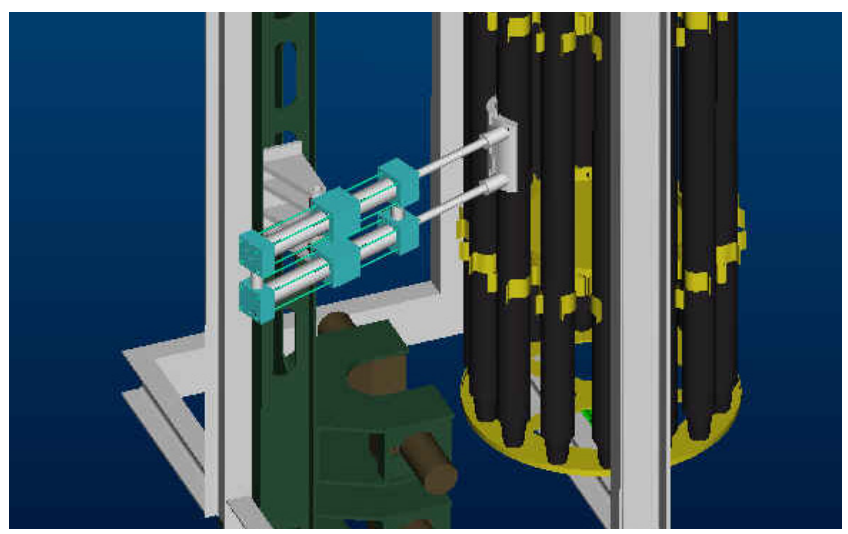

Fig. 16. Schematic of the 3 DOF manipulator (jacks for rotation and gripper opening/closing are not shown).

\section{B. Manipulators with $2 \mathrm{DOF}$}

The first solution of 2 DOF manipulator consists of a gripper sliding on a rail fixed to the frame between two columns, Fig. 17. It presents noteworthy stiffness, with a direct fall-off on precision aspects.

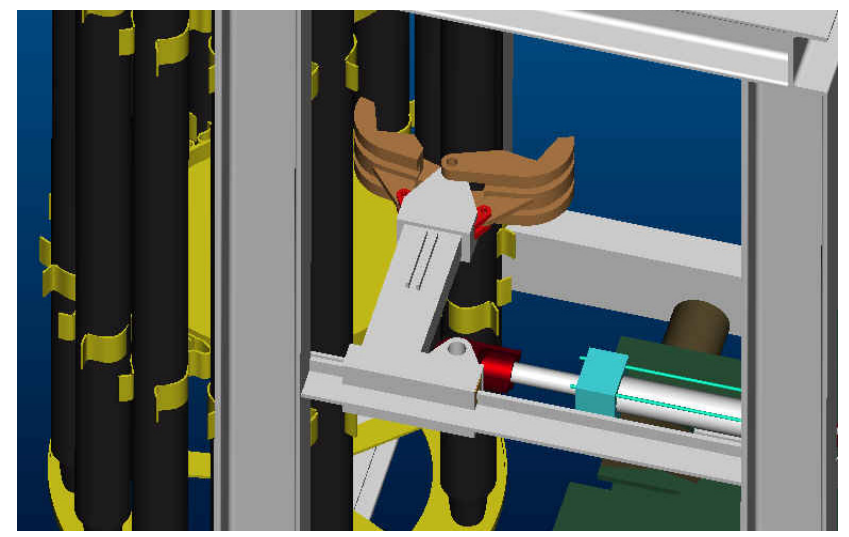

Fig. 17. Schematic of the enhanced cartesian manipulator.

This manipulator has two degrees of freedom, but, because it works exploiting the final and initial position sets, it requires an on-off type control. The first degree of freedom is a translational on a slider running on the milled sides of an offthe-shelf beam, screwed to the structural frame of the drilling unit.

A drawback is the wear of the gibs that cause clearance with misalignment errors. The pick point of the rod is in the plane passing through the buffer axis and the axis of the drilling machine, because the length of the manipulator (perpendicular to the rail) is constant.

The extreme positions correspond, respectively, the first, to the grasp point for the rods from/to the buffer, the other, to the axis of the drilling machine. The gripper is composed by two fingers that close contemporary on the rod (the motion is transmitted by two beams pushed-pulled by a jack) and have an internal shape allowing self-centering.

During the phases of loading and unloading of the rods, the gripper has to open till the fingers are aligned, to avoid interference with the other rods and with the drilling head; the rod is, in fact, moved through a plane passing between the axis of the rotating buffer and the axis of the driller.

A second solution, which has been finally adopted, consists of a three-fingers unit having only one pivoting finger in order to simplify its design and realization. This unit is moved forth and back by an electric linear actuator (with trapezoid screw); the pivoting finger rotates by means of a double-effect (on-off) hydraulic jack, Fig. 18, Fig. 19, and Fig. 20.
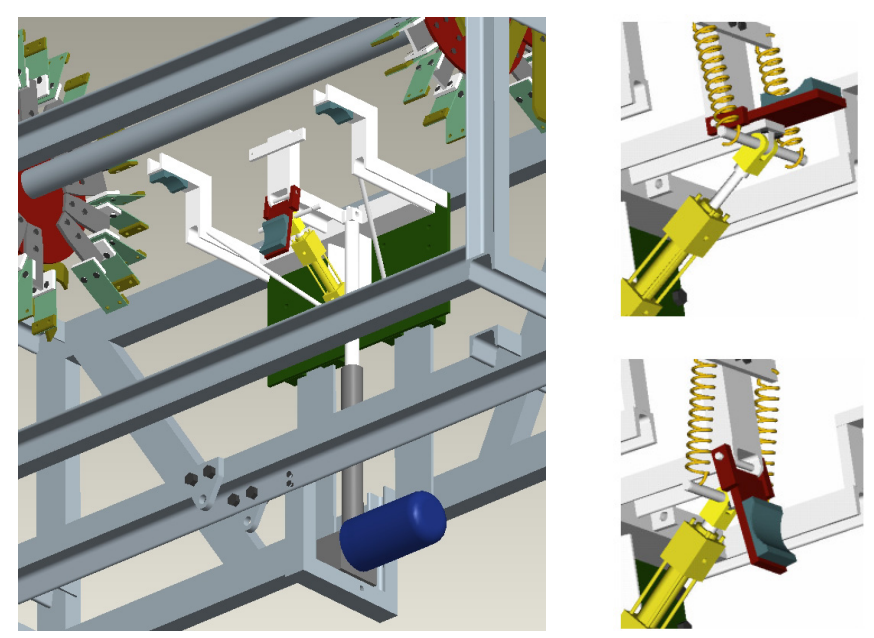

Fig. 18. The manipulator mounted on the drilling frame; the guiding rails and the motor for its linear translation are also illustrated. On the right: close/open positions of the gripper.

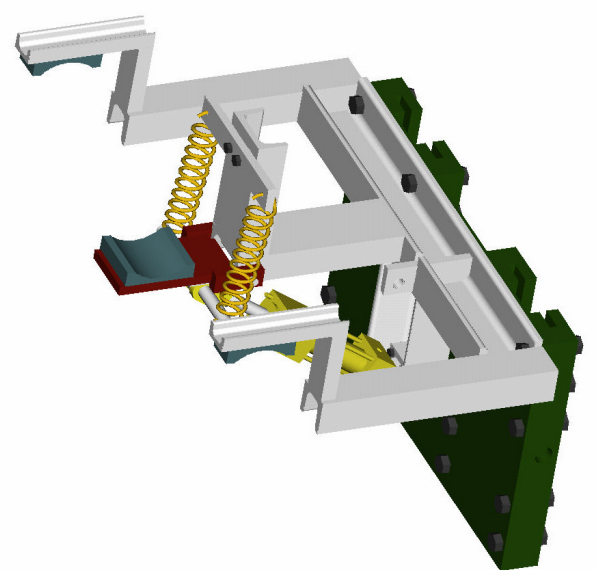

Fig. 19. The manipulator: note the sliding guides on the rear and the springs granting firm grasp even with no pressure inside the jack (for safety reasons).

The linear actuator has a speed: $10 \mathrm{~mm} / \mathrm{s}$, maximum thrust: $2500 \mathrm{~N}$, and it is equipped with three set-position switches.

It might seem that two positions would be enough, the rod being moved from the buffer to the drilling axis and back; 


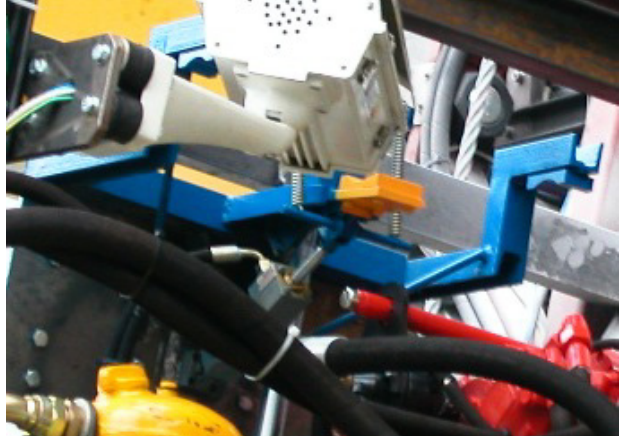

Fig. 20. The real manipulator. On the foreground, one of the CCD cameras needed for tasks monitoring.

actually, because the gripper has only one rotating finger, it is necessary to go ahead with the fixed fingers of about $25 \mathrm{~mm}$, in order to avoid collisions among them and the rod (see sequence in Fig. 21).

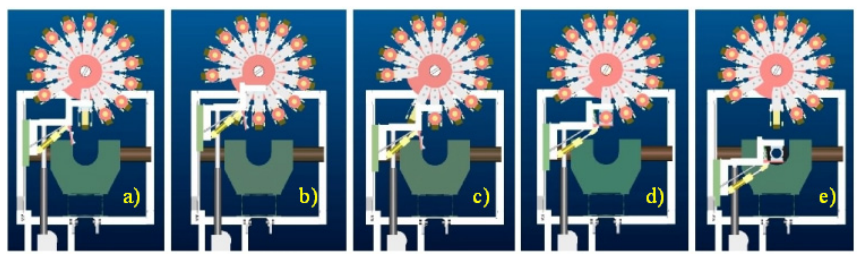

Fig. 21. Key steps for transferring a rod from the buffer to the drilling axis: a) the gripper is in its rest position; b) the gripper moves $25 \mathrm{~mm}$ up while the buffer rotates; c) the gripper goes down till the fixed fingers touch the rod; d) the pivoting finger presses against on the rod; e) the gripper moves down and reach the driller axis.

\section{CONCLUSION}

The sharing of knowledge from scientific literature, expertise and experience of the Research Centers, and of the industrial firms involved in the Roboclimber Project were used to derive the technologies and methodologies for the system design and development. A multidisciplinary approach was adopted to solve the problem with large use of mathematical modeling, computer simulation, digital mock-up and virtual reality testing tools in order to compare and evaluate several conceptual solutions and single out those maximizing the overall system performances. Mechanical and control architecture have been conceived simultaneously while considering modularity aspects and lifecycle issues. The enduser has been included in the design loop at every level in order to assure the system effectiveness and work suitability.

Future developments regard the drilling unit and the rods manipulator. For the driller an improvement will be the capability of folding up the rail along which the drilling head runs: this will avoid the disassembly of the drilling system out of the Roboclimber before its transportation on trucks as, when the rail is folded, the maximum height will be adequate to standard road tunnels size.

The rod manipulator will be modified as it has been observed, after severe trials, that dust and small rocks tend to block the good sliding of the fingers on the prismatic rails; the change will focus on the rails that will be circular and on the actuator for the translation which will be made of two paired hydraulic cylinders, instead of the present electric one, to get enhanced force and reliability, and three fixed positions managing without need of control switches.

It is worthwhile noting that the appreciated characteristics of autonomously operating of Roboclimber and of its drilling system in non-structured environment suggest their use in extra-terrestrial exploration: in fact, Roboclimber's efficiency in various terrain conditions and its good mobility performances assure the successful accomplishment of unmanned tasks even in regions not earlier mapped.

\section{REFERENCES}

[1] R. Molfino, M. Armada, F. Cepolina and M. Zoppi, "Roboclimber, the 3 tons spider," Industrial Robot Journal, Vol. 32, No. 2, 2005, to be published.

[2] M. Moronti, M. Sanguineti, M. Zoppi, and R. Molfino, "Roboclimber: proposal for online gait planning," 7th International Conference on Climbing and Walking Robots CLAWAR04, Madrid, Spain, 2004.

[3] P. Anthoine, M. Armada, S. Carosio, P. Comacchio, F. Cepolina, T. Klopf, F. Martin, R. C. Michelini, R. M. Molfino, S. Nabulsi, R. P. Razzoli, E. Rizzi, L. Steinicke, R. Zannini, and M. Zoppi, "A FourLegged Climbing Robot for Rocky Slope Consolidation and Monitoring," Int. World Automation Congress WAC2004, ISBN 1889335-20-7, Seville, Spain, 2004.

[4] M. Zoppi, S. Sgarbi, R. M. Molfino, and L. Bruzzone, "Equilibrium analysis of quasi-static, multi-roped walking robots," Int. Conf. Climbing and Walking robots and the support technology for mobile machines CLAWAR03, ISBN 1-86058-409-8, Catania, Italy, September, 17-19, 2003, pp. 259-266.

[5] P. Anthoine, M. Armada, S. Carosio, P. Comacchio, F. Cepolina, P. González , T. Klopf , F. Martin, R. C. Michelini, R. M. Molfino, S. Nabulsi, R. P. Razzoli, E. Rizzi, L. Steinicke, R. Zannini, M. Zoppi, "Roboclimber," ASER03, 1st International Workshop on Advances in Service Robotics, Bardolino, Italy, ISBN 3-8167-6268-9, March 13-15, 2003.

[6] G. Acaccia, L. E. Bruzzone, R. C. Michelini, R. M. Molfino, R. P. Razzoli, "A tethered climbing robot for firming up high-steepness rocky walls," Proc. of the 6th IAS Intl. Conf. on Intelligent Autonomous Systems, edited by E. Pagello, F. Groen, T. Arai, R. Dillmann, A. Stentz, published by IOS press, Amsterdam, ISBN 158603078 7, Italy, Venice, July, 25-27, 2000, pp. 307-312.

[7] S. Nabulsi, H. Montes, M Armada, "Roboclimber: Control System architecture," 7th International Conference on Climbing and Walking Robots CLAWAR04, Madrid, Spain, 22-24 September 2004.

[8] S. Nabulsi, M. A. Armada, and P. González de Santos, "Control Architecture for a Four-Legged Hydraulically Actuated Robot," 13th International Symposium on Measurements and Control in Robotics (ISMCR'03), Ponencia, Madrid, 11-12 December 2003, pp.169-174.

[9] H. Montes, S. Nabulsi, M. Armada, and V. Sanchez, "Design and implementation of force sensor for Roboclimber," Proceedings of Int. Conf. of Climbing and Walking Robots, CLAWAR 2004. Madrid, Spain, 22-24 September 2004

[10] M. A. Armada, S. Nabulsi, "Climbing strategies for remote maneuverability of Roboclimber," Proceedings of the 35th International Symposium on Robotics, Paris, France, 23-26 March 2004.

[11] L. Steinicke, C. Dal Zot, T. Benoist, "A System for Monitoring and Controlling a Climbing and Walking Robot for Landslide Consolidation," IARP Int. Workshop Robotics and mechanical Assistance in Humanitarian Demining and Similar Risky Interventions, Brussels-Leuven, Belgium, 16-18 June 2004. 